\title{
Blazhko modulation in first-overtone RR Lyrae stars from the OGLE Collection
}

\author{
Henryka Netzel ${ }^{1,2 \star}$, Radosław Smolec ${ }^{2 \star \star}$, Igor Soszyński ${ }^{1}$, and OGLE Team \\ ${ }^{1}$ Warsaw University, Astronomical Observatory, Al. Ujazdowskie 4, 00-478 Warsaw, Poland \\ ${ }^{2}$ Nicolaus Copernicus Astronomical Centre, Polish Academy of Sciences, Bartycka 18, 00-716 Warsaw, \\ Poland
}

\begin{abstract}
We present results of the first comprehensive study of the Blazhko effect in first-overtone RR Lyrae stars from the Galactic bulge based on the Optical Gravitational Lensing Experiment data.
\end{abstract}

\section{Introduction}

RR Lyrae stars are classically pulsating stars. Most of them are simple single-periodic radial pulsators, which pulsate either in the radial fundamental mode (RRab type) or in the radial first overtone (RRc type). We also know double-mode RR Lyrae stars pulsating simultaneously in the radial fundamental mode and the first overtone (RRd type) or in the fundamental mode and the second overtone, but the latter are scarce.

Despite their astrophysical importance and relative simplicity there are still unsolved problems concerning RR Lyrae stars. One of the most stubborn problems is the Blazhko effect, which is a quasi-periodic modulation of pulsation amplitude and/or phase. It was discovered by Sergey Blazhko in 1907 ([3]), who observed changes in moments of maximum light of RW Dra. Since its discovery, the Blazhko effect has lacked a satisfactory explanation.

In these proceedings we present a first comprehensive study of the Blazhko effect in first-overtone RR Lyrae stars from the Galactic bulge. As a data source, we used data from the fourth phase of the Optical Gravitational Lensing Experiment (OGLE; [15]). OGLE regularly monitors the brightness of more than a billion sources. Excellent data quality together with numerous observations and long time base are perfect for a search of Blazhko effect in RR Lyrae stars.

\section{Data and method}

In the OGLE collection of variable stars there are more than 38000 RR Lyrae stars from the Galactic bulge. 10826 among them are of RRc type ([13]). Because of the numerous input sample, we developed an automatic algorithm for analysis and selection of Blazhko RRc stars. The algorithm uses Fourier analysis and consecutive prewhitening method. In principle, the Blazhko effect manifests itself in the power spectrum as equally spaced multiplets centered on the main frequency and its

\footnotetext{
${ }^{\star}$ henia@netzel.pl

$\star \star$ smolec@camk.edu.pl
} 


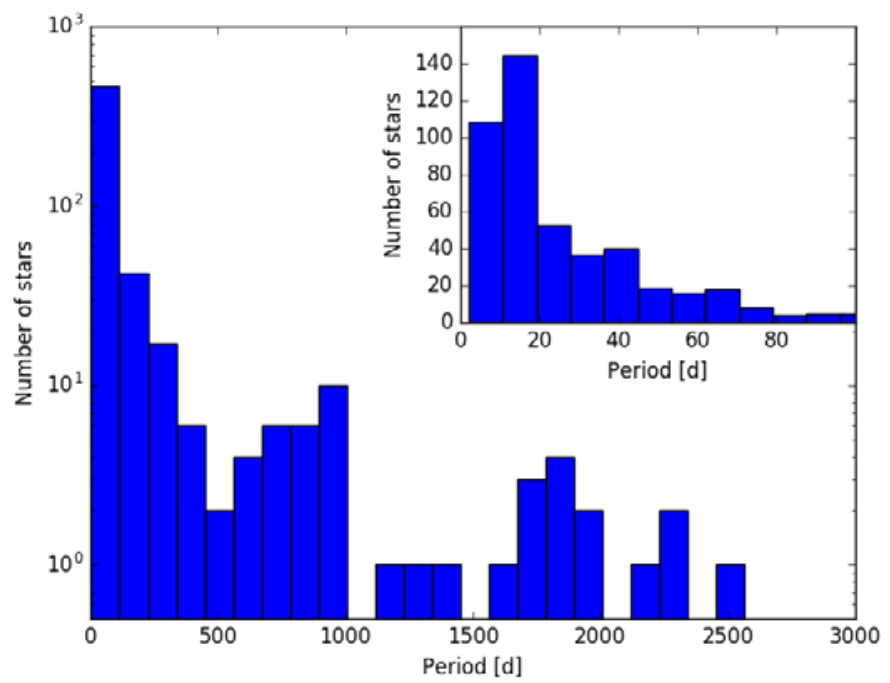

Figure 1. Histogram of Blazhko periods for RRc stars. In the top right corner of the plot we include a zoom-in for the short-period range. Note that the number of stars in the zoom-in is plotted in a linear scale, whereas the whole distribution is plotted in a logarithmic scale.

harmonics. Separation between the multiplet components corresponds to the modulation frequency. In ground-based photometry we usually detect triplets or doublets. The algorithm searched for these characteristic features in the power spectrum. If the algorithm detected at least two sidepeaks with the same separation, it considered a star to be a Blazhko candidate. The algorithm selected 3066 candidates, which constitutes 28 per cent of the input sample. However, many RRc stars exhibit period changes, which manifest themselves in power spectra as a remnant power in the position of the first overtone after the prewhihtening. This remnant power together with its annual aliases can lead to misclassification of a star as a long-period Blazhko variable. To verify the candidates we visually inspected the power spectra of all 3066 candidates. Stars which passed the visual inspection were all analysed manually.

\section{Results}

The analysis resulted in 573 Blazhko RRc stars, which constitutes 5.3 per cent of the input sample. In 384 stars we detected triplets (BL2), which constitute 67 per cent of the Blazhko sample. Doublets were detected in 189 stars (BL1), which constitute 33 per cent of the Blazhko sample. For a full table of Blazhko stars together with their properties, see Netzel et. al. (in prep.). The distribution of Blazhko periods in stars from our sample is presented in Figure 1. In the top right corner of this figure we present a zoom-in for periods shorter than 100 days. Note that the full distribution is plotted in a logarithmic scale, whereas the zoom-in is plotted in a linear scale. Stars with short periods of modulation are the most numerous. The shortest detected Blazhko period is 2.23 days for a BL1 star. Among BL2 stars the shortest period is 4.9 days. The longest period of the Blazhko effect depends on the time base of observations. OGLE-IV data allow on average to detect modulation with periods up to 1000 days. This is the reason for a sudden drop in the number of stars in Figure 1. However, 


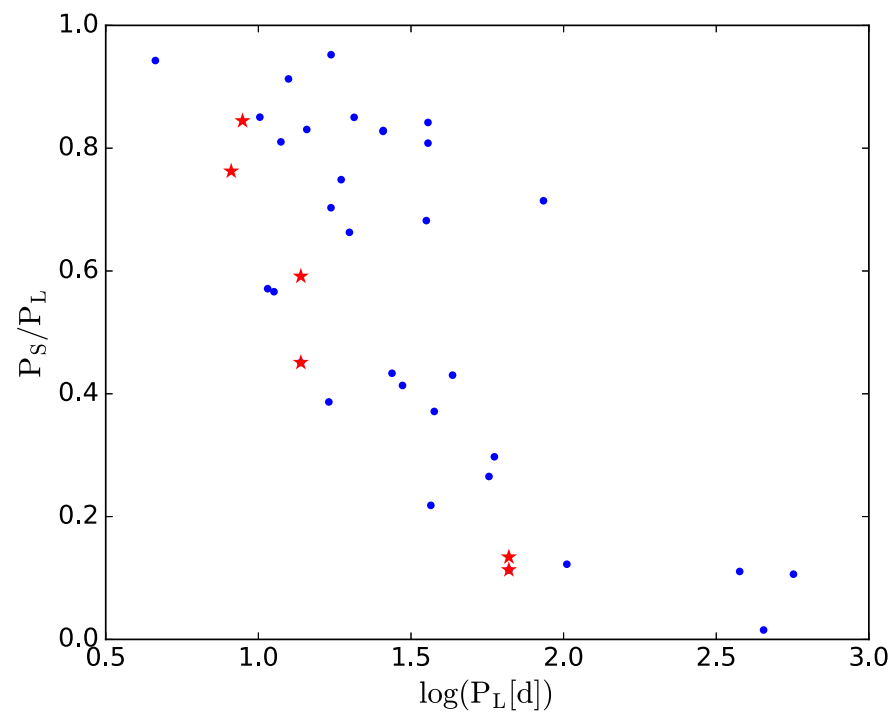

Figure 2. Relation between the ratio of shorter to longer Blazhko period versus longer Blazhko period for stars with multi-periodic Blazhko effect. Stars with two Blazhko periods are marked with blue dots. Stars with three Blazhko periods are marked with red asterisks.

combining OGLE data from the previous phases of the experiment allowed us to detect a few stars with modulation periods longer than this limit. The longest modulation period detected is 2477 days.

In 32 stars from our sample we detected two Blazhko periods. Three Blazhko periods were detected in 2 stars from our sample. Blazhko periods of these stars are presented in Figure 2 in the form of a "Petersen diagram", i.e. we plot the ratio of the shorter to longer modulation period versus the longer period. The majority of these stars have short periods of modulations (below 50 days). Only in three stars do we observe a significantly longer period. Observed period ratios cover a wide range, and no particular grouping that could correspond e.g. to a ratio of small integer numbers is observed.

Besides the main pulsation frequency, its harmonics and Blazhko sidepeaks, additional significant signals were detected in dozens of stars. In Figure 3 we present the Petersen diagram for already known double-mode RR Lyrae stars. On top of these stars, we plot Blazhko stars in which we detected additional signals (orange symbols). Many of these stars fall into the known groups: several stars fall into 0.61 and 0.68 groups, four stars are close to the RRd sequence and might be anomalous RRd stars ([11, 12]; see also Smolec et al. in these proceedings). There is another numerous group on the Petersen diagram with high period ratios (above 0.9). In these stars we observe additional signal with frequency very close to the first-overtone frequency. It can be either a non-radial mode with period close to the main pulsation period or a manifestation of another Blazhko modulation (but since we did not detect other sidepeaks, the star was not classified as a Blazhko variable).

One of the most intriguing phenomena discovered in Blazhko RRab stars is period doubling, which manifests as alternating minima and maxima in the light curve. In the power spectrum, it manifests as signals close to half-integer frequencies. This phenomenon was detected for the first time in RR Lyr from Kepler data ([5]), and later studies showed that it affects many modulated RRab stars (see, e.g., [2, 14]). It motivated a new model explaining the Blazhko effect ([4]). Period doubling 


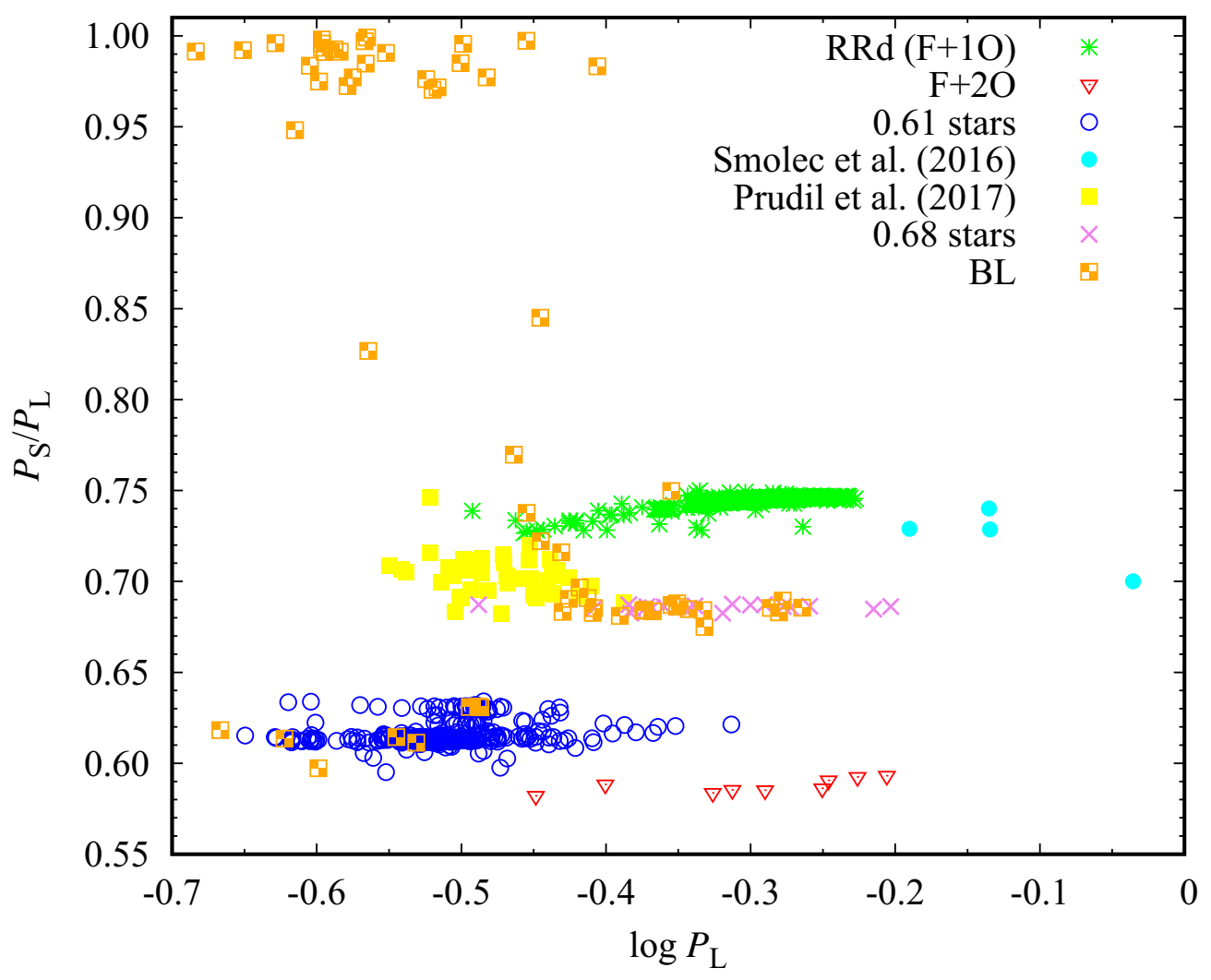

Figure 3. Petersen diagram for the known groups of double-periodic RR Lyrae stars. RRd stars are marked with green asterisks, fundamental mode and second overtone stars are marked with red triangles, first overtone ones with a shorter-period non-radial mode stars forming a period ratio 0.61 are marked with blue circles ([8]), first overtone and long-periodic variability forming a period ratio 0.68 are marked with pink crosses ([7]), RR Lyrae stars with short-period signal are marked with yellow squares ([9]) and RRab stars with another shortperiod signal are marked with blue filled circles ([10]). Blazhko stars with additional signals from our sample are marked with orange symbols.

was not detected in Blazhko RRc stars. During manual analysis of our Blazhko candidates we did not find any significant signal close to half-integer frequencies. In order to detect this effect we followed a procedure outlined in [1], and averaged the power spectra of all Blazhko stars in our sample, after prewhitening with pulsation and modulation components. All spectra were scaled, so the main pulsation frequency of each star corresponds to 1 . The average spectrum is presented in Figure 4. In the case of stars in which we observed a remnant signal at the position of the main pulsation frequency due to e.g. a period change, we removed the effect using a time-dependent prewhitening method ([6]). Use of this method resulted in a sharp drop visible in Figure 4 at $f / f_{1 \mathrm{O}}=1$. If Blazhko RRc stars exhibit the period doubling effect, in Figure 4 we should see signals around values 0.5 and 1.5 , which is not the case. We note however that detection of the period doubling effect in the groundbased data is challenging and our results do not eliminate the possibility that it plays a role also in Blazhko RRc stars. 


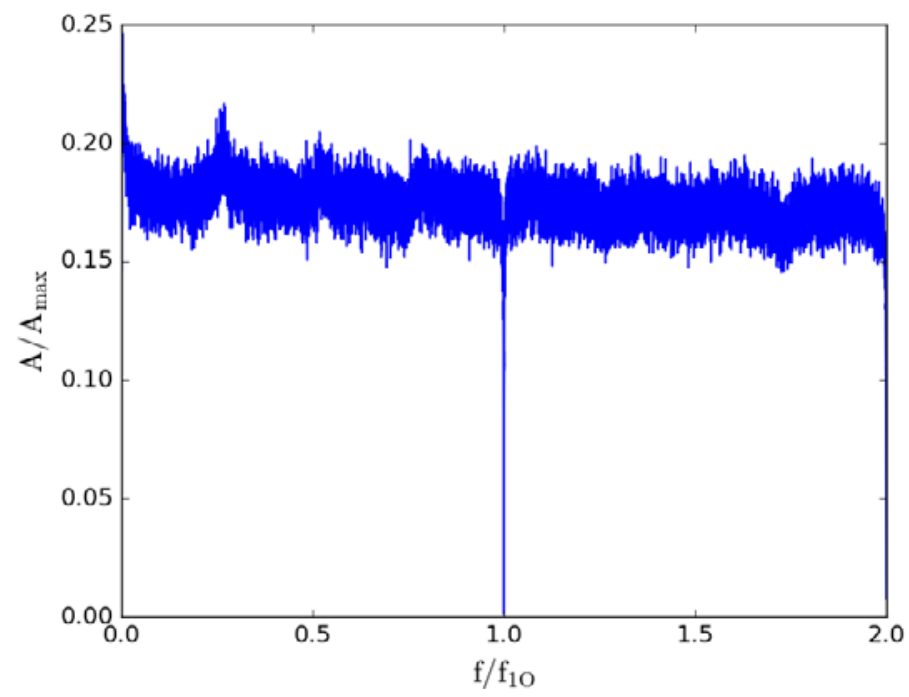

Figure 4. Averge spectrum of all Blazhko stars from our sample. We removed the period change present in some stars using time-dependent prewhitening. Spectra are scaled, so 1 on the horizontal axis corresponds to the frequency of the first overtone.

Full results of this study will be published elsewhere (Netzel et al., in preparation).

Acknowledgments: This research is supported by the Ministry of Science and Higher Education through grant no. 0192/DIA/2016/45 and by the Polish National Science Centre through grant no. DEC-2015/17/B/ST9/03421. $\mathrm{HN}$ is grateful to the organizers for their financial support.

\section{References}

[1] Alcock, C., Alves, D. R., Becker, A., et al., ApJ, 598, 597 (2003)

[2] Benkő, P. E., Szabó, R., Molnár, L., \& Kolláth, Z., ApJS, 213, 13 (2014)

[3] Blazko, S., AN, 175, 325 (1907)

[4] Buchler, J. R., \& Kolláth, Z., ApJ, 731, 24 (2011)

[5] Kolenberg, K., Szabó, R., Kurtz, D. W., et al., ApJ, 713, L198 (2010)

[6] Moskalik, P., Smolec, R., Kolenberg, K., et al., MNRAS, 447, 2348 (2015)

[7] Netzel, H., Smolec, R., \& Dziembowski, W., MNRAS, 451, L25 (2015)

[8] Netzel, H., Smolec, R., \& Moskalik, P., MNRAS, 453, 2022 (2015)

[9] Prudil, Z., Smolec, R., Skarka, M., \& Netzel, H., MNRAS, 465, 4074 (2017)

[10] Smolec, R., Prudil, Z., Skarka, M., \& Bakowska, K., MNRAS, 461, 2934 (2016)

[11] Smolec, R., Soszyński, I., \& Udalski, A., MNRAS, 447, 3753 (2015)

[12] Soszyński, I., Smolec, R., \& Dziembowski, W. A., MNRAS, 463, 1332 (2016)

[13] Soszyński, I., Udalski, A., Szymański, M.K., et al., Acta Astron., 64, 177 (2014)

[14] Szabó, R., Benkő, J. M., Paparó M., et al., A\&A, 570, A100 (2014)

[15] Udalski, A., Szymański, M.K., \& Szymański, G., Acta Astron., 65, 1 (2015) 